\title{
Effect of environmental factors and sex difference on physiological responses of the exotic red swamp crayfish, Procambarus clarkii, in Egypt.
}

\author{
Victoria G. A. Nicola ; Omaima M. Mustafa; Samir M. H. Beltagi \\ and Hany N.Yousef. \\ Department of Biological and Geological Sciences, Faculty of Education, Ain \\ Shams University, Egypt.
}

\begin{abstract}
$\mathrm{T}$ he current study was conducted to investigate the effect of environmental factors and sex difference on the physiological status of the exotic red swamp crayfish (Procambarus clarkii) in Egypt along four successive seasons during the period from 2005 to 2006. Animals were collected from El-Mansourya canal at Giza governorate. Water and sediments were collected from three different aquatic habitats in order to determine the least polluted water quality region for collecting specimens used in the current study . These sites were: El-Mansourya canal (site I), El-Mouhit drain (site II) and Bany Magdoul canal (site III). During the four seasons, measurements of physicochemical characteristics of water and sediments revealed elevated annual concentrations for ammonia, nitrates, nitrites, in addition to high chlorosity in site II and heavy contamination with iron, copper, manganese, zinc and aluminum in site III; whereas site I was the least polluted and possesses the most transparent water with lowest electrical conductivity than the other sites. Lowest water temperature was recorded during winter and the highest one was during summer; while at the same season no wide difference has been found among the three habitats. Biochemical analysis of the main edible parts of crayfish inhabiting El-Mansourya canal (site I) revealed that males showed little rise in their muscle water content but females exhibited significant higher lipid content almost the year round. No marked changes were observed in the protein content in both sexes. Females also showed more glycogen accumulation in their abdominal muscles but lack statistical significance except during spring . In both sexes, highest levels for water content were reported during winter and for lipid during spring while for glycogen were during autumn. The lowest content for lipid was during autumn and for glycogen during winter for both sexes. The results indicate that seasonal changes in biochemical composition of edible parts of both sexes could reflect different physiological processes related to sex difference as an expression of animal adaptive response, an ecophysiological adaptation, to habitat quality and environmental conditions along the four successive seasons in Egypt.
\end{abstract}

Key words : Crayfish, Procambarus clarkii, habitat quality, sex difference, seasonal variations, biochemical parameters. 


\section{INTRODUCTION}

Procambarus clarkii is considered to be the most ecologically plastic freshwater species in the Family Cambaridae. This crayfish is a polytrophic, migratory, oxyconformer, eurythermic and exotic invertebrate animal (Mauro and Thompson,1984). It is able to tolerate slightly saline water, which is unusual for a crayfish and also being able to tolerate dry spells of up to four months . Red swamp crayfish is long-lived species. The average lifetime of $P$. clarkii is 5 years, while some individuals have reached ages over 6 years in nature. Red swamp crayfish has been accidentally introduced into the Egyptian freshwater systems in the early 1980's (Ibrahim et al.,1995and 1996). Due to its high migratory ability, resistance to environmental changes and high ability to tolerate variable temperature as well as low water quality (Johnson and Avault,1982; Payette and McGaw, 2003: Zanatto et al.,2004). It has been greatly spread without control, multiplied its numbers and rapidly expanded in most of Egyptian freshwater ecosystems (Emam \& Khalil, 1995; Ibrahim et al.,1996). The wide spread and the abundance of this species in most of the governorates of lower and upper Egypt (Saad and Emam,1998) induced the interest of many workers to investigate different activities in crayfish such as reproduction, behaviour, growth, survival as well as physiological, ecological, biological and economical importance and its geographic distribution (Meyer et al.,1991; Sayed, 2002; Hammond et al.,2006).

Environmental factors such as temperature, $\mathrm{pH}$, salinity, dissolved oxygen, ammonia, nitrate, nitrite, and heavy metals, have optimum levels suitable for the growth of aquatic animals . Changing levels of these physicochemical parameters of the aquatic habitats affect the physiology, distribution, growth and survival of the freshwater crayfish (Austin,1995b; Liu et al., 1995; Shakir and Ibrahim, 1998; Tolba, 1999; Abdall and El-Zawahry, 2001; Rowe et al., 2001; Abd El-Monem, 2002).

Many physiological functions including oxygen consumption, feeding and enzyme reaction rates are temperature dependent .Crayfish are eurithermic that can tolerate temperature variations through behavioral adjustment, resistance and physiological functions (Carmona-Osalde et al., 2004).

In Australia, Jones (1995) found that the best growth of Cherax quadricarinatus was achieved at 28 and $24{ }^{\circ} \mathrm{C}$.Under laboratory conditions, Habashy (2004a) found that survival and growth rate of P. clarkii was $90 \%$ at $20^{\circ} \mathrm{C}$ and the highest weight gain was obtained at the same temperature, whereas below $20^{\circ} \mathrm{C}$ and above $30{ }^{\circ} \mathrm{C} \mathrm{P}$. clarkii showed higher mortality percentage (Abdel - Wahed, 2006).

Water $\mathrm{pH}$ has a direct effect on organisms as well as an indirect effect on the toxicity of certain pollutants in water (Alabaster and Lioyd,1982). Although Procambarus can tolerate a wide range of $\mathrm{pH}$, Huner (1988) stated that it should be in the range of 7.2- 8.5. The $\mathrm{pH}$ values at different sites along the River Nile 
of Egypt were always fluctuating between 7.2 and 9.2 under the effect of seasonal variations (Mubarak, 1997 ; Abd El-Monem , 2002).

Ammonia $\left(\mathrm{NH}_{3}\right)$ is highly soluble in water and exists in two forms : a molecular form associated with water and in un- ionized form as $\mathrm{NH}_{4}{ }^{+}$. The extent of association or ionization is dependent on the temperature and $\mathrm{pH}$. It may also be toxic to aquatic life (ASTM, 2002). Only the un-ionized fraction of ammonia is poisonus. This fraction increases with rising of temperature and $\mathrm{pH}$ value. The acute toxicity $\left(96-\mathrm{hr} \mathrm{LC}_{50}\right.$ ) of ammonia to juvenile $P$. clarkii was 2.65 $\mathrm{mg} \mathrm{NH}_{3}-\mathrm{N} / \mathrm{L}$ (Liu et al.,1995). The severe biological effect of nitrite on crustacean species was indicated by Rouse and Yeh (1995); since under the effect of nitrite, crustacean hemocyanin changes to methoglobin causing hypoxia and cyanosis .Although dissolved oxygen (DO) is required for the survival and growth of many aquatic organisms, the freshwater crayfish can adapt to hypoxic environment (Mauro and Thompson, 1984; Ingerson and Geddes, 1995). DO should be maintained above $3 \mathrm{ppm}$. for optimal crawfish production (Huner, 1988).

Water chlorosity at different sites of the River Nile ranged between $0.03 \mathrm{~g} / 1$ during spring and $0.38 \mathrm{~g} / \mathrm{l}$ during autumn (Mubarak,1997); while those recorded by Abd El-Monem (2002) were 25 and $71 \mathrm{ppm}$ for water from clean and polluted canal, respectively.

Certain heavy metals may be either beneficial or toxic depending on their concentrations (APHA,1985).The increased metal levels not only diminish water and sediment quality but also can accumulate in the bodies of animals and plants and transfer along food chains resulting in health consequences in humans (Rainbow,1997).In water, copper, iron, manganese, and zinc have been classified as essential heavy metals that help in the growth and reproduction of aquatic life (Rizkalla and Abo-Donia, 1996), while DÍaz-Mayans et al.(1986) and Madigosky et al (1991) reported that crustaceans as well, P. clarkii, tend to accumulate metals in their tissues. Copper is involved in the functioning of a variety of proteins including the crustacean respiratory protein haemocyanin (Dallinger,1977). Although iron is vital for most living organisms, iron and copper are potentially harmful in high concentrations (Maranhao et al., 1995). Alikhan et al.(1990) observed a general relationship between crayfish tissue metal concentrations and those in water and sediments, but insignificant differences between males and females at each sampling site were recorded. Crayfish were used as bio-indicator of trace metal pollution in the aquatic habitats because they tend to accumulate metals in their tissues (Meyer et al.,1991); while in 1993, Mwangi and Alikhan found that zinc stores in various tissues of Cambarus bartoni and $\mathrm{Cu}$ content in muscles, gonads and exoskeleton but remained unaffected by the presence of either $\mathrm{Cd}$ or Ni. The levels of proteins, carbohydrates and lipids are an expression of an animal's adaptive characteristics and its strategies for adaptation against many biotic functions and abiotic factors that can strongly affect the biochemistry and physiology of 
decapod crustaceans (Company and Sardà, 1998; Rosa and Nunes, 2003a \& b ; Vinagre et al.,2007).

The biochemical composition of abdominal muscles of the crayfish $P$. clarkii either collected from clean or polluted habitats or subjected to certain heavy metals were investigated by Huner et al.(1988), Torreblanca et al. (1992)and El Mossalami and Emara (1999). Fernandes et al. (1995) observed an increase in lipid content during spring followed by a decrease during summer to levels maintained through the winter period, while seasonal changes in protein and carbohydrate contents were relatively limited. Mona et al.(2000) and Mubarak (2001) demonstrated that male P. clarkii were higher than females in concern to moisture, protein and lipid contents. Hepatopancreas and muscles of the crustacean Parastacus varicosus did not show any significant difference between males and females in concern to glycogen, protein, total lipids and total cholesterol, while the same parameters revealed significant differences in the same sex along the four seasons (Silva-Castiglioni et al.,2007).Seasonal variations in biochemical composition of $P$. defosus were recently investigated by Buckup et al.(2008).

Metallothionein like protein induction by exposure to trace metals has been identified in several decapod crustaceans (Roesijadi, 1994 ; Rainbow, 2002). This group of metal-proteins is believed to provide a protective role against toxic effects of metals by binding and sequestering these metals (Roesijadi, 1992; Abdall and El-Zawahry, 2001 ; Khan and Nugegoda, 2007). The hypothesis of the current investigation postulates that environmental factors and water quality could induce variable effects in physiological status of the exotic red swamp crayfish in both sexes along the year and accordingly, different physiological responses related to sex difference could change the biochemical composition of certain edible parts of this species in its new environment in Egypt. So, it is of interest here to investigate the effect of certain abiotic factors (habitat quality and seasonal variations) and biotic factor (sex difference) on the biochemical composition of the main edible muscles of $P$. clarkii in order to obtain more information about physiological adaptation of this animal beside the nutritive value of its flesh that is considered a cheaper source of animal protein for human consumption .

\section{MATERIALS AND METHODS}

STUDY REGIONS: The three aquatic habitats found in Kirdasa, Giza governorate are: El-Mansourya canal (site I) which receives little domestic and agricultural effluents. It was preferable for the current study as it was the least polluted area after carrying the physicochemical measurements; El-Muhit drain(siteII) containing polluted water with large quantities of sewage and agricultural effluents and Bany Magdoul canal (siteIII) which is filled with more polluted water. It receives the discharge of car mechanician workshops, 
car tires fixing workshops and cement bricks' workshops, in addition to the domestic effluents.

WATER AND SEDIMENT ANALYSIS: In order to characterize the habitat quality and seasonal variations, certain physicochemical parameters comprising water temperature, $\mathrm{pH}$ and dissolved oxygen content were measured in addition to water transparency, $\mathrm{EC}, \mathrm{Cl}, \mathrm{NH}_{3}, \mathrm{NO}_{3}$ and $\mathrm{NO}_{2}$. During the period from 2005 to 2006 , temperature was measured at the time of sampling with a mercury thermometer. Transparency of water was measured by using Secchi disk $(30 \mathrm{~cm}$ diameter).Water $\mathrm{pH}$ was measured by using a glass electrode $\mathrm{pH}$-meter. Electrical conductivity of water (EC) was measured using EC-meter and expressed as $(\mu / \mathrm{cm})$. Dissolved Oxygen (DO) was measured by the DO probe and expressed as $(\mathrm{mg} / \mathrm{l})$. Chlorides $(\mathrm{Cl})$ in water were determined volumetrically and ammonia $\left(\mathrm{NH}_{3}\right)$ was determined colourimetrically according to ASTM (2002). Nitrates $\left(\mathrm{NO}_{3}\right)$ and nitrites $\left(\mathrm{NO}_{2}\right)$ in water were determined according to APHA(1992). The heavy metals analyzed in water and sediments were iron, copper, zinc, manganese and aluminum in the three aquatic habitats during the four seasons.

\section{Biochemical Study:}

Biochemical analysis was carried out on abdominal muscle samples of mature specimens measuring $\geq 3.5 \mathrm{~cm}$ carapace length (Huner et al., 1988)of red swamp crayfish of both sexes inhabiting El-Mansourya canal (siteI) representing the least polluted areas in the current study. Abdominal muscle water content was estimated as described by Sokolov (1966). Total lipid content (ethyl-ether soluble fraction) was estimated gravimetrically as demonstrated by Tietz et al. (1970).

Total protein content in the tissue extract was estimated photometrically using the Biuret method as described by Wooton (1964). The glycogen content in abdominal muscle extract was estimated photometrically according to the method of Carroll et al.( 1955).

STATISTICAL ANALYSIS: The biological data of the current study were tabulated, computed and analyzed statistically applying the student $t$ test . The level of significance was calculated and considered to be significant when $\mathrm{P}^{*}$ is less than 0.05 , highly significant when $\mathrm{P}^{* *}$ is less than 0.01 and very highly significant when $\mathrm{P}^{* * *}$ is less than 0.001 .

\section{RESULTS AND DISCUSSION}

Data in Tables (1- 7) show seasonal values and annual means of certain physicochemical parameters of water beside certain heavy metals of water and sediment in the three sites of collection. The results revealed that the three study regions vary greatly in their water habitat quality. El-Mansourya canal (site I) possesses the most transparent water with lowest electrical conductivity as compared to sites II and III along the four seasons. Therefore, site I was a preferable region for specimen collection used in the current study as it was the least polluted area compared to the other two sites ( II \&III). Site II exhibited 
elevated levels of $\mathrm{NH}_{3}, \mathrm{NO}_{3}, \mathrm{NO}_{2}, \mathrm{Cl}$ and $\mathrm{EC}$ with the minimum level of DO (Tables1-3); while water in site III were highly contaminated with iron, copper, manganese, zinc and aluminum . Similarly, sediment showed maximum levels of most heavy metals (Tables 4-7).

Table (1): Values of physico-chemical characteristics of water samples collected from ElMansourya canal (siteI), El-Muhit drain (site II) and Bany Magdoul canal (site III) along four successive seasons.

\begin{tabular}{|c|c|c|c|c|c|}
\hline \multirow{2}{*}{ Item } & \multirow{2}{*}{ Site } & \multicolumn{4}{|c|}{ Season } \\
\hline & & Winter & Spring & Summer & Autumn \\
\hline \multirow{3}{*}{ 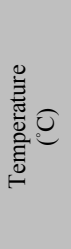 } & I & 18.5 & 27.0 & 31.0 & 23.0 \\
\hline & II & 19.0 & 28.0 & 32.0 & 24.0 \\
\hline & III & 18.0 & 27.5 & 30.0 & 23.0 \\
\hline \multirow{3}{*}{ 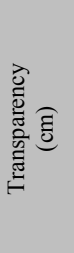 } & I & 52.5 & 70.0 & 65.0 & 62.5 \\
\hline & II & 30.0 & 35.0 & 35.0 & 32.5 \\
\hline & III & 25.0 & 30.0 & 30.0 & 27.5 \\
\hline \multirow{3}{*}{ 吾 } & I & 7.3 & 7.5 & 8.0 & 8.0 \\
\hline & II & 6.8 & 7.1 & 8.2 & 8.1 \\
\hline & III & 7.1 & 7.2 & 8.7 & 8.2 \\
\hline \multirow{3}{*}{ 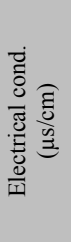 } & I & 745.0 & 530.0 & 511.0 & 625.0 \\
\hline & II & 1330.0 & 1184.0 & 1940.0 & 1758.0 \\
\hline & III & 756.0 & 554.0 & 745.0 & 805.0 \\
\hline \multirow{3}{*}{ 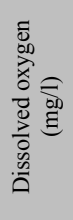 } & I & 2.2 & 4.0 & 4.4 & 4.0 \\
\hline & II & 2.3 & 0.9 & 0.9 & 1.5 \\
\hline & III & 3.8 & 0.3 & 4.6 & 3.9 \\
\hline
\end{tabular}


Table (2): Values of Chlorosity, Ammonia, Nitrates and Nitrites of water samples collected from El-Mansourya canal (site I), El-Muhit drain (site II) and Bany Magdoul canal (site III) along four successive seasons.

\begin{tabular}{|c|c|c|c|c|c|c|}
\hline & \multirow{2}{*}{ Item } & \multirow{2}{*}{ Site } & \multicolumn{4}{|c|}{ Season } \\
\hline & & & Winter & Spring & Summer & Autumn \\
\hline \multirow{3}{*}{ 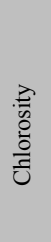 } & \multirow{3}{*}{ 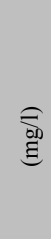 } & I & 75.30 & 47.87 & 44.13 & 54.47 \\
\hline & & II & 174.89 & 167.55 & 320.53 & 285.96 \\
\hline & & III & 77.73 & 52.66 & 78.97 & 86.24 \\
\hline & \multirow{3}{*}{$\widehat{\overparen{D}}$} & I & 0.319 & 0.214 & 0.414 & 0.289 \\
\hline & & II & 0.853 & 0.768 & 0.924 & 0.861 \\
\hline & & III & 0.256 & 0.241 & 0.297 & 0.251 \\
\hline \multirow{3}{*}{ 总 } & \multirow{3}{*}{$\widehat{\widehat{D D D}}$} & I & 1.32 & 1.70 & 0.90 & 1.10 \\
\hline & & II & 7.47 & 7.50 & 3.70 & 3.10 \\
\hline & & III & 1.41 & 1.40 & 0.84 & 0.91 \\
\hline \multirow{3}{*}{ 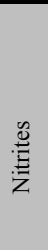 } & \multirow{3}{*}{$\widehat{\widehat{D D D}}$} & I & 0.248 & 0.285 & 0.315 & 0.259 \\
\hline & & II & 0.466 & 0.510 & 0.640 & 0.484 \\
\hline & & III & 0.247 & 0.250 & 0.275 & 0.256 \\
\hline
\end{tabular}


Table (3): Annual means of physico-chemical characteristics of water samples collected from El-Mansourya canal (site I), El-Muhit drain (site II) and Bany Magdoul canal (site III).

\begin{tabular}{|c|c|c|c|c|}
\hline \multirow{2}{*}{\multicolumn{2}{|c|}{ Item }} & \multicolumn{3}{|c|}{ Site } \\
\hline & & I & II & III \\
\hline \multirow{2}{*}{$\begin{array}{c}\text { Temperature } \\
\left({ }^{\circ} \mathrm{C}\right)\end{array}$} & Mean & 24.87 & 25.75 & 24.62 \\
\hline & $\%$ change & & $3.54 \%$ & $1.00 \%$ \\
\hline \multirow[t]{2}{*}{ Transparency } & Mean & 62.5 & 33.1 & 28.1 \\
\hline & $\%$ change & & $7.00 \%$ & $5.00 \%$ \\
\hline \multirow{2}{*}{$\mathrm{pH}$} & Mean & 7.70 & 7.55 & 7.80 \\
\hline & $\%$ change & & $1.95 \%$ & $1.30 \%$ \\
\hline \multirow[t]{2}{*}{ Electrical cond. } & Mean & 602.75 & 1553 & 715 \\
\hline & $\%$ change & & $157.65 \%$ & $18.62 \%$ \\
\hline \multirow[t]{2}{*}{ Dissolved oxygen } & Mean & 3.65 & 1.40 & 3.15 \\
\hline & $\%$ change & & $61.64 \%$ & $14.00 \%$ \\
\hline \multirow[t]{2}{*}{ Chlorosity } & Mean & 55.44 & 237.23 & 73.90 \\
\hline & $\%$ change & & $327.90 \%$ & $33.29 \%$ \\
\hline \multirow{2}{*}{$\begin{array}{c}\text { Ammonia } \\
(\mathrm{mg} / \mathrm{l})\end{array}$} & Mean & 0.31 & 0.85 & 0.26 \\
\hline & $\%$ change & & $174.19 \%$ & $16.13 \%$ \\
\hline \multirow{2}{*}{$\begin{array}{l}\text { Nitrates } \\
(\mathrm{mg} / \mathrm{l})\end{array}$} & Mean & 1.26 & 5.44 & 1.14 \\
\hline & $\%$ change & & $331.75 \%$ & $9.52 \%$ \\
\hline \multirow[t]{2}{*}{ Nitrites } & Mean & 0.28 & 0.53 & 0.26 \\
\hline & $\%$ change & & $89.29 \%$ & $7.14 \%$ \\
\hline
\end{tabular}


Table (4): Heavy metal concentrations of water samples collected from El Mansourya canal (site I), El Muhit drain (site II) and Bany Magdoul canal (site III) along four successive seasons.

\begin{tabular}{|c|c|c|c|c|c|c|}
\hline \multirow{2}{*}{\multicolumn{2}{|c|}{ Metal }} & \multirow{2}{*}{ Site } & \multicolumn{4}{|c|}{ Season } \\
\hline & & & Winter & Spring & Summer & Autumn \\
\hline & \multirow{3}{*}{ 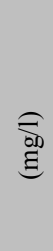 } & I & 0.1398 & 0.1329 & 0.4217 & $<0.0200$ \\
\hline & & II & 0.1591 & 0.2430 & 0.3200 & 0.1745 \\
\hline & & III & 0.2218 & 0.3439 & 0.5938 & 5.227 \\
\hline & \multirow{3}{*}{ 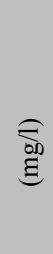 } & I & $<0.0100$ & $<0.0200$ & 0.0460 & $<0.0300$ \\
\hline & & II & $<0.0170$ & $<0.0200$ & 0.0511 & 0.0300 \\
\hline & & III & 0.0271 & $<0.0200$ & 0.0837 & 0.2698 \\
\hline & \multirow{3}{*}{$\widehat{\overparen{b 0}}$} & I & 0.0368 & 0.1321 & 0.1555 & 0.0321 \\
\hline & & II & 0.0220 & 0.0917 & 0.2144 & 0.1601 \\
\hline & & III & 0.1769 & 0.2768 & 0.0803 & 0.4945 \\
\hline \multirow{3}{*}{$\stackrel{\mathscr{N}}{\mathrm{N}}$} & \multirow{3}{*}{$\begin{array}{l}\widehat{\widehat{D 0}} \\
\hat{\text { ह }}\end{array}$} & I & 0.0743 & 0.0426 & 0.1273 & 0.0048 \\
\hline & & II & 0.0674 & 0.0224 & 0.0905 & 0.0300 \\
\hline & & III & 0.0920 & 0.1251 & 0.1472 & 0.2025 \\
\hline \multirow{3}{*}{ 志 } & \multirow{3}{*}{$\widehat{\overparen{b 0}}$} & I & 0.2166 & $<0.1000$ & $<0.1000$ & $<0.1000$ \\
\hline & & II & 0.1586 & 0.2338 & 0.2207 & 0.2115 \\
\hline & & III & 0.1484 & 0.6931 & 0.3630 & 0.8593 \\
\hline
\end{tabular}


Table (5): Annual averages of heavy metal concentrations of water samples collected from El Mansourya canal (site I), El Muhit drain (site II) and Bany Magdoul canal (site III).

\begin{tabular}{|c|c|c|c|c|}
\hline \multirow{2}{*}{\multicolumn{2}{|c|}{ Metal }} & \multicolumn{3}{|c|}{ Site } \\
\hline & & I & II & III \\
\hline \multirow{2}{*}{$\begin{array}{l}\text { Iron } \\
(\mathrm{mg} / \mathrm{l})\end{array}$} & Mean & 0.179 & 0.224 & 1.597 \\
\hline & $\%$ change & & $25.14 \%$ & $792.18 \%$ \\
\hline \multirow{2}{*}{$\begin{array}{l}\text { Copper } \\
(\mathrm{mg} / \mathrm{l})\end{array}$} & Mean & 0.027 & 0.030 & 0.100 \\
\hline & $\%$ change & & $11.11 \%$ & $270.37 \%$ \\
\hline Manganese & Mean & 0.089 & 0.122 & 0.257 \\
\hline$(\mathrm{mg} / \mathrm{l})$ & $\%$ change & & $37.08 \%$ & $188.76 \%$ \\
\hline \multirow{2}{*}{$\begin{array}{l}\text { Zinc } \\
(\mathrm{mg} / \mathrm{l})\end{array}$} & Mean & 0.062 & 0.053 & 0.142 \\
\hline & $\%$ change & & $14.52 \%$ & $129.03 \%$ \\
\hline \multirow{2}{*}{$\begin{array}{l}\text { Aluminum } \\
(\mathrm{mg} / \mathrm{l})\end{array}$} & Mean & 0.129 & 0.206 & 0.516 \\
\hline & $\%$ change & & $59.69 \%$ & $300.00 \%$ \\
\hline
\end{tabular}


Table (6): Heavy metal concentrations of sediment samples collected from El Mansourya canal (site I), El Muhit drain (site II) and Bany Magdoul canal (site III) along four successive seasons.

\begin{tabular}{|c|c|c|c|c|c|c|}
\hline \multirow{2}{*}{\multicolumn{2}{|c|}{ Metal }} & \multirow{2}{*}{ Site } & \multicolumn{4}{|c|}{ Season } \\
\hline & & & Winter & Spring & Summer & Autumn \\
\hline & \multirow{3}{*}{$e^{\circ}$} & I & 1.6700 & 1.5050 & 1.6100 & 2.3760 \\
\hline & & II & 1.9600 & 1.6400 & 3.2600 & 2.5180 \\
\hline & & III & 3.9900 & 4.5000 & 3.9600 & 3.6720 \\
\hline & \multirow{3}{*}{ de } & I & 0.0023 & 0.0320 & 0.0026 & 0.0110 \\
\hline & & II & 0.0022 & 0.0300 & 0.0070 & 0.0100 \\
\hline & & III & 0.0048 & 0.0730 & 0.0100 & 0.0240 \\
\hline \multirow{3}{*}{ 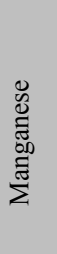 } & \multirow{3}{*}{ de } & I & 0.0130 & 0.0114 & 0.0091 & 0.0350 \\
\hline & & II & 0.0200 & 0.0250 & 0.0670 & 0.0390 \\
\hline & & III & 0.0930 & 0.0750 & 0.0630 & 0.1200 \\
\hline \multirow{3}{*}{ 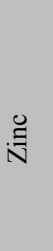 } & \multirow{3}{*}{$\stackrel{2}{d}$} & I & 0.0470 & 0.0064 & 0.0031 & 0.0080 \\
\hline & & II & 0.0490 & 0.0133 & 0.0079 & 0.0420 \\
\hline & & III & 0.0480 & 0.0179 & 0.0200 & 0.0090 \\
\hline \multirow{3}{*}{ 音 } & \multirow{3}{*}{ 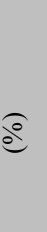 } & I & 1.3600 & 1.3800 & 1.3200 & 2.0020 \\
\hline & & II & 0.8900 & 1.6000 & 2.8500 & 1.6420 \\
\hline & & III & 4.0100 & 4.2500 & 4.5300 & 3.3210 \\
\hline
\end{tabular}


Table (7): Annual averages of heavy metal concentrations of sediment samples collected from El Mansourya canal (site I), El Muhit drain (site II) and Bany Magdoul canal (site III) along four successive seasons.

\begin{tabular}{|c|c|c|c|c|}
\hline \multirow{2}{*}{\multicolumn{2}{|c|}{ Metal }} & \multicolumn{3}{|c|}{ Site } \\
\hline & & I & II & III \\
\hline \multirow{2}{*}{$\begin{array}{l}\text { Iron } \\
(\%)\end{array}$} & Mean & 1.790 & 2.345 & 4.031 \\
\hline & $\%$ change & & $31.01 \%$ & $125.19 \%$ \\
\hline \multirow{2}{*}{$\begin{array}{c}\text { Copper } \\
(\%)\end{array}$} & Mean & 0.012 & 0.012 & 0.028 \\
\hline & $\%$ change & & $0.00 \%$ & $133.33 \%$ \\
\hline \multirow{2}{*}{$\begin{array}{c}\text { Manganese } \\
(\%)\end{array}$} & Mean & 0.017 & 0.038 & 0.088 \\
\hline & $\%$ change & & $123.53 \%$ & $417.65 \%$ \\
\hline \multirow{2}{*}{$\begin{array}{l}\text { Zinc } \\
(\%)\end{array}$} & Mean & 0.016 & 0.028 & 0.024 \\
\hline & $\%$ change & & $75.00 \%$ & $50.00^{-0} \%$ \\
\hline \multirow{2}{*}{$\begin{array}{c}\text { Aluminum } \\
(\%)\end{array}$} & Mean & 1.516 & 1.746 & 4.028 \\
\hline & $\%$ change & & $15.17 \%$ & $165.70 \%$ \\
\hline
\end{tabular}

Low DO concentration in water of site II was not a great problem for the crayfish since it can adapt to hypoxic environment by physiological responses to low oxygen supply as indicated by Mauro and Thompson (1984) and Ingerson and Geddes (1995). They reported that these responses include increasing respiratory water flow over the gills and increasing the affinity of hemocyanin for oxygen with a low metabolic demand by tissues. Analysis of water and sediments during the four seasons (Tables1and3) revealed that lowest water temperature during winter and the highest one during summer; while no clear difference has been found between water temperature in the three aquatic habitats at the same season.

In the current study, the data in Tables (8-11) were for biochemical analysis of the abdominal muscle samples of both male and female crayfish indicated that certain environmental factors in Egypt could induce marked changes in the metabolic profile of the collected specimens during the four successive seasons .

Abdominal muscle water content in crayfish inhabiting site I are within similar values recorded by Huner et al.(1988) for both Astacus and 
Procambarus species (79\%- 85\%) and also by Huner et al. (1990) on Astacus species only, where the muscle water content ranged from $75-84 \%$ in both sexes depending on the molt stage and reproductive state of the animal . Similar results were also obtained by El-Mossallami and Emara (1999),but lower values were recorded by Mona et al. (1999) and Mubarak (2001) on the same species in Egypt.

Table (8): The water content of abdominal muscles ( $\mathrm{g} / 100 \mathrm{~g}$ wet wt.) of male and female Procambarus clarkii collected from El Mansourya canal along four successive seasons .

\begin{tabular}{|c|c|c|c|c|c|}
\hline Seasons & Parameters & Males & № & Females & № \\
\hline \multirow{4}{*}{ Winter } & $\begin{array}{c}\text { Range } \\
\text { Minimum-maximum }\end{array}$ & $80.19-83.99$ & \multirow{4}{*}{7} & $77.41-82.55$ & \multirow{4}{*}{7} \\
\hline & Mean \pm SE & $82.30 \pm 0.47$ & & $79.42 \pm 0.60$ & \\
\hline & P Level & & & $\mathrm{P}<0.01 * *$ & \\
\hline & $\%$ Change & & & $3.50 \%$ & \\
\hline \multirow{4}{*}{ Sprng } & Range & $80.00-81.71$ & \multirow{4}{*}{7} & $75.48-79.18$ & \multirow{4}{*}{8} \\
\hline & Mean \pm SE & $80.72 \pm 0.25$ & & $77.16 \pm 0.42$ & \\
\hline & P Level & & & $\mathrm{P}<0.001 * * *$ & \\
\hline & \%Change & & & $4.41 \%$ & \\
\hline \multirow{4}{*}{ Summer } & Range & $78.68-83.54$ & \multirow{4}{*}{6} & $75.09-81.23$ & \multirow{4}{*}{6} \\
\hline & Mean $\pm \mathrm{SE}$ & $80.15 \pm 0.75$ & & $77.48 \pm 0.94$ & \\
\hline & P Level & & & $\mathrm{P}>0.05$ & \\
\hline & \%Change & & & $3.33 \%$ & \\
\hline \multirow{4}{*}{ Autumn } & Range & $79.27-82.43$ & \multirow{4}{*}{6} & $73.44-79.86$ & \multirow{4}{*}{5} \\
\hline & Mean \pm SE & $80.83 \pm 0.50$ & & $76.64 \pm 1.23$ & \\
\hline & P Level & & & $\mathrm{P}<0.01^{* *}$ & \\
\hline & $\%$ Change & & & $5.18 \%$ & \\
\hline
\end{tabular}

It was of interest to denote that under seasonal variations in Egypt, the current data for muscle biochemical composition could reflect sex individual differences in physiological responses of crayfish against environmental conditions during the time of experimentation. However, data in (Table 8) indicate that male 
crayfish exhibited higher water content than females collected from the same site; where the fall in female muscle water content was statistically significant along the four successive seasons except slight difference during summer season as compared to male crayfish.This was in partial agreement with the results obtained by Mona et al. (1999) and Mubarak (2001) .

Mean values for total lipid content in the abdominal muscles of both sexes in site 1 ranged from 0.95 to $4.85 \mathrm{~g} / 100 \mathrm{~g}$ dry wt (Table 9).This is in agreement with the results obtained by Mona et al.(1999) and Mubarak (2001) for Procambarus clarkii but exceeded the range recorded by El-Mossallami and Emara (1999) on the same species in Egypt.

Table (9): The total lipid content of abdominal muscles (g/100g dry wt.)of male and female Procambarus clarkii collected from El Mansourya canal along four successive seasons.

\begin{tabular}{|c|c|c|c|c|c|}
\hline Seasons & Parameters & Males & № & Females & № \\
\hline \multirow{4}{*}{ Winter } & $\begin{array}{c}\text { Range } \\
\text { Minimum-maximum }\end{array}$ & $2.10-2.94$ & \multirow{4}{*}{5} & $2.27-2.82$ & \multirow{4}{*}{6} \\
\hline & Mean $\pm \mathrm{SE}$ & $2.36 \pm 0.15$ & & $2.59 \pm 0.08$ & \\
\hline & P Level & & & $\mathrm{P}>0.05$ & \\
\hline & $\%$ Change & & & $9.75 \%$ & \\
\hline \multirow{4}{*}{ Spring } & Range & $3.32-4.58$ & \multirow{4}{*}{8} & $4.47-5.26$ & \multirow{4}{*}{8} \\
\hline & Mean $\pm \mathrm{SE}$ & $3.94 \pm 0.15$ & & $4.85 \pm 0.09$ & \\
\hline & P Level & & & $\mathrm{P}<0.001 * * *$ & \\
\hline & $\%$ Change & & & $23.10 \%$ & \\
\hline \multirow{4}{*}{ Summer } & Range & $1.72-2.10$ & \multirow{4}{*}{6} & $2.02-2.36$ & \multirow{4}{*}{6} \\
\hline & Mean $\pm \mathrm{SE}$ & $1.86 \pm 0.06$ & & $2.16 \pm 0.05$ & \\
\hline & P Level & & & $\mathrm{P}<0.01 * *$ & \\
\hline & $\%$ Change & & & $16.13 \%$ & \\
\hline \multirow{4}{*}{ Autumn } & Range & $0.74-1.28$ & \multirow{4}{*}{8} & $1.66-2.19$ & \multirow{4}{*}{8} \\
\hline & Mean \pm SE & $0.95 \pm 0.06$ & & $1.91 \pm 0.06$ & \\
\hline & P Level & & & $\mathrm{P}<0.001 * * *$ & \\
\hline & $\%$ Change & & & $101.05 \%$ & \\
\hline
\end{tabular}

It is evident that females exhibited higher lipid content in their abdominal muscles than males at the same site (Table 9). This increase was 
statistically significant along most of the year. Similar results were recorded by Fernandes et al. (1995) where the total lipids and triglycerides contents are much lower and did not oscillate in as wide range as in females. Conversely, the present results contradict those obtained by Mona et al.(1999) and Mubarak (2001)where they observed that, the abdominal muscles of male P.clarkii contain more lipids than females. The high level of lipids in females may be attributed to the fact that females require large amount of energy for egg production or due to the ability of female to recover from $\mathrm{Cu}$-induced impairment slightly faster than males, suggesting a more efficient mechanism for detoxifying copper. Active mobilization of lipid reserves from storage tissues to the gonads for the build up of gametes in crustacean species was reported by Teshima and Kanazawa (1983) and Millamena and Pascual (1990).It is clear that maximum lipid concentrations in abdominal muscles were recorded during spring and then decreased gradually during summer until they reached minimum levels during autumn in either males or females. These results agree with those of Fernandes et al.(1995) and Abd El-Monem (2002) on the same species. The storage of lipids during spring may be used as fuel for the synthesis of reproductive products that is confirmed by the decrease in total lipids, in both sexes, during the following seasons until reaching minimum level in autumn that represents the end of the main reproductive period.

The total protein content of abdominal muscles of both male and female crayfish lies in the range of 11.14 to $14.59 \mathrm{~g} / 100 \mathrm{~g}$ wet wt(Table 10). These results comply with those obtained by El-Mossallami and Emara(1999)on Procambarus clarkii in Egypt but were lower than that obtained by Mona et al. (1999) and Mubarak (2001) on the same species. Also our results are below the range recorded by Huner et al.(1990) on Astacus astacus. There was no marked difference between both sexes in total protein content of abdominal muscles during the four seasons. These results are in agreement with those obtained by Fernandes et al. (1995) who found that the protein profiles of soft tissues of $P$. clarkii were similar in males and females throughout the year except for a significant peak in November/January period. Close results on Parastacus varicosus were obtained by Silva-Castigliona et al.(2007).

The current results for protein content in both sexes at autumn showed slight but insignificant rise. This may be due to the beginning of a storage process as reported after Ibrahim et al. (1996) as this animal stores protein as a transient nutritional reserve after the utilization for most of the stored lipids for the requirements of reproduction. This hypothesis is supported by the results of Hubbard et al.(1986) and Fernandes et al.(1995)on the same species .

Although females of P.clarkii in the current study exhibited higher glycogen content in their abdominal muscles than males most of the year, but the recorded seasonal changes lack statistical significance except during spring (Table11). This is in agreement with that of Silva-Castigliona et al.(2007) who found that hepatopancreas and muscles of Parastacus varicosus did not show any 
significant difference between male and female glycogen, protein, total lipids and total cholesterol, while the same parameters revealed significant differences in the same sex along the four seasons. In the present study, glycogen content in abdominal muscles of males and females reached minimum values in winter and peaked in autumn.

Table (10): The total protein content of abdominal muscles (g/100g wet wt.) of male and female Procambarus clarkii collected from El Mansourya canal along four successive seasons .

\begin{tabular}{|c|c|c|c|c|c|}
\hline Seasons & Parameters & Males & № & Females & № \\
\hline \multirow{4}{*}{ Winter } & $\begin{array}{c}\text { Range } \\
\text { Minimum-maximum }\end{array}$ & $9.64-14.98$ & \multirow{4}{*}{8} & $10.54-16.41$ & \multirow{4}{*}{6} \\
\hline & Mean \pm SE & $11.84 \pm 0.60$ & & $12.83 \pm 1.09$ & \\
\hline & P Level & & & $\mathrm{P}>0.05$ & \\
\hline & $\%$ Change & & & $8.36 \% \uparrow$ & \\
\hline \multirow{4}{*}{ Spring } & Range & $10.32-11.65$ & \multirow{4}{*}{6} & $10.80-12.13$ & \multirow{4}{*}{8} \\
\hline & Mean $\pm \mathrm{SE}$ & $11.14 \pm 0.18$ & & $11.28 \pm 0.18$ & \\
\hline & P Level & & & $\mathrm{P}>0.05$ & \\
\hline & $\%$ Change & & & $1.26 \% \uparrow$ & \\
\hline \multirow{4}{*}{ Summer } & Range & $11.24-11.56$ & \multirow{4}{*}{5} & $11.16-13.29$ & \multirow{4}{*}{6} \\
\hline & Mean $\pm \mathrm{SE}$ & $12.78 \pm 0.57$ & & $12.22 \pm 0.34$ & \\
\hline & P Level & & & $\mathrm{P}>0.05$ & \\
\hline & $\%$ Change & & & $4.38 \% \downarrow$ & \\
\hline \multirow{4}{*}{ Autumn } & Range & $11.09-17.39$ & \multirow{4}{*}{6} & $10.50-17.42$ & \multirow{4}{*}{8} \\
\hline & Mean $\pm \mathrm{SE}$ & $14.59 \pm 0.92$ & & $13.78 \pm 0.93$ & \\
\hline & P Level & & & $\mathrm{P}>0.05$ & \\
\hline & $\%$ Change & & & $5.55 \%$ & \\
\hline
\end{tabular}


The current seasonal variations in muscle glycogen content could be explained according to Hagerman et al.(1990) and Baden et al.(1994); where the lowest values obtained in the winter correspond to the period of the year with the highest percentage of empty stomachs. This was supported by Schirf et al. (1987) who reported that food deprivation in P. clarkii resulted in a significant decrease in muscle carbohydrate. Similar results were obtained by Rosa and Nunes ( 2003a) on deep-sea decapod crustaceans since the glycogen content in abdominal muscles of these animals reached the lowest values in winter. Also, Dutra et al.(2007) recorded that in both sexes of the crustacean Hyalella curvispina, glycogen was stored during summer and utilized especially during winter and autumn; while Fernandes et al. (1995) reported that, seasonal changes in carbohydrate content were relatively limited in soft tissues of $P$. clarkii.

Table(11): The total glycogen content of abdominal muscles (mg/100g wet wt.) of male and female Procambarus clarkii collected from El Mansourya canal along four successive seasons.

\begin{tabular}{|c|c|c|c|c|c|}
\hline Seasons & Parameters & Males & № & Females & № \\
\hline \multirow{4}{*}{ Winter } & $\begin{array}{c}\text { Range } \\
\text { Minimum-maximum }\end{array}$ & $51.61-91.01$ & \multirow{4}{*}{5} & $63.06-139.33$ & \multirow{4}{*}{6} \\
\hline & Mean \pm SE & $79.20 \pm 7.71$ & & $92.44 \pm 10.57$ & \\
\hline & P Level & & & $\mathrm{P}>0.005$ & \\
\hline & $\%$ Change & & & $16.72 \%$ & \\
\hline \multirow{4}{*}{ Spring } & Range & $159.14-189.46$ & \multirow{4}{*}{8} & $182.62-200.73$ & \multirow{4}{*}{7} \\
\hline & Mean \pm SE & $171.53 \pm 3.24$ & & $188.26 \pm 2.96$ & \\
\hline & P Level & & & $\mathrm{P}<0.01 * *$ & \\
\hline & $\%$ Change & & & $9.75 \%$ & \\
\hline \multirow{4}{*}{ Summer } & Range & $154.60-171.18$ & \multirow{4}{*}{6} & $158.50-188.88$ & \multirow{4}{*}{6} \\
\hline & Mean \pm SE & $163.36 \pm 2.69$ & & $167.88 \pm 4.75$ & \\
\hline & P Level & & & $\mathrm{P}>0.05$ & \\
\hline & $\%$ Change & & & $2.77 \%$ & \\
\hline \multirow{4}{*}{ Autumn } & Range & $146.14-300.71$ & \multirow{4}{*}{5} & $212.00-378.08$ & \multirow{4}{*}{7} \\
\hline & Mean \pm SE & $221.43 \pm 27.97$ & & $259.89 \pm 21.91$ & \\
\hline & P Level & & & $\mathrm{P}>0.05$ & \\
\hline & $\%$ Change & & & $17.37 \%$ & \\
\hline
\end{tabular}


Seasonal variations in biochemical composition of P.clarkii's edible parts in the current study showed highest levels for water during winter and for lipid during spring and for glycogen during autumn in both sexes. While lowest content for lipid was recorded during autumn and for glycogen was during winter in both sexes. This fall in muscle glycogen at this season could be referred to its use by the crayfish during the time of fasting or food shortage as indicated by Buckup et al.( 2008) in both male and female Parastacus defosus .

From the current study it can be concluded that seasonal changes in the biochemical parameters of edible parts in male and female red swamp crayfish (P.clarkii) could reflect different physiological processes related to sex difference as an expression of animal adaptive response(ecophysiological adaptation) to habitat quality and environmental conditions along the four successive seasons in Egypt.

\section{REFERENCES}

Abdall, A. T. and El-Zawahry, E. I.(2001). Bioaccumulation of lead and copper in the freshwater crayfish Procambarus clarkii, spectrometric and biochemical studies. J. Egypt. Acad. Soc. Environ. Develop., 2 (2): 39-106.

Abd El-Monem, M. A.(2002). Effect of water quality and some parasites on some biological aspects of fresh water crayfish at Sharkia governorate. Ph.D. Thesis. Zool. Dept. Fac. of Sci., Zagazig Univ., Egypt.

Abdel-Wahed, H. R.(2006). Biological and physiological studies on the freshwater crayfish Procambarus clarkii. Ph.D. Thesis. Zool. Dept. Girls Collage for Art, Sci. and Edu., Ain Shams Univ., Egypt.

Alabaster, J. S and Lioyd, R. (1982). Water quality criteria for freshwater fish. $2^{\text {nd }}$ ed. Butterworth Scientific, London.

Alcorlo, A.; Otero, M.; Crehuet, M.; Baltanás, A. and Montes, C. (2006). The use of the red swamp crayfish (Procambarus clarkii,Girard) as indicator of the bioavailability of heavy metals in environmental monitoring in the River Guadiamar (SW,Spain). Sci. Tot .Environ., 366: $380-390$. 
Ali Khan, M. A.; Bagatto, G. and Zia, S. (1990). The crayfish as a biological indicator of aquatic contamination by heavy metals .Water Res., 24(9):1069-1076.

American Public Health Association (APHA, 1985). Standard methods for the examination of water and wastewater. $16^{\text {th }}$ ed., Washington., D. C.

American Public Health Association (APHA, 1992). Standard methods for the examination of water and waste water. $18^{\text {th }}$ ed., Washington., D.C.

American Society for Testing and Materials (ASTM, 2002). Annual book of ASTM standards, section 11 Water and Environmental Technology., 11(1)., ASTM International., West Conshohocken.

Austin, C. M. (1995b). Effect of temperature and salinity on the survival and growth of juvenile red claw (Cherax quadricarinatus). Freshwat. Crayfish., 10: 419-426.

Baden, S.P.; Depledge, M.H. and Hagerman, L. (1994). Glycogen depletion and altered copper and manganese handling in Nephrops norvegicns (L) following starvation and exposure to hypoxia. Mar. Environ. Res., 39 (1-4): 342-343.

Buckup, L.; Dutra, B.K.;Ribarcki,F.P.; Fernandes, F.A.; Noro, C.K.; Olveira, G.T. and Vinagre, A.S. (2008). Seasonal variations in the biochemical composition of the crayfish Parastacus defossus (Crustacea, Decapoda) in its natural environment. Comp. Biochem. Physiol., A. 149: 59-67.

Carmona-Osalde, C. C.; Rodriguez-Serna, M. ; Olvera-Novoa, M. A. and Gutierrez-Yurrita, P. J. (2004). Gonadal development, spawning, growth and survival of the crayfish Procambarus llamasi at three different water temperatures. Aquacult., 232: 305-316.

Carroll, N. V.; Longley, R. W. and Roe, J. H. (1955). The determination of glycogen in liver and muscle by use of anthrone reagent. J. Biol. Chem., 220: 583-593. 
Company, J.B. and Sardà, F. (1998). Metabolic rates and energy content of deep-sea decapod crustaceans in the western Mediterranean Sea. Deep-Sea Res., I 45:1861-1880.

Dallinger, R. (1977). The flow of copper through a terrestrial food chain III. Selection of an optimum copper diet by isopods. Oceologia., 30: 273277.

DÍaz-Mayans,J.;Hermández,F.;Medina，J.; Del Ramo，J.and torreblanca，A. (1986). Cadmium accumulation in the crayfish , Procambarus clarkii , using graphite furnace atomic absorption spectrometry. Arch. Environ. Contam. Toxicol.,37:722-729.

Dutra, B. K.; Santos, R. B; Bueno, A. A. P. and Oliveira, G. T. (2007). Seasonal variations in the biochemical composition and lipoperoxidation of Hyalella curvispina (Crustacea, Amphipoda). Comp. Biochem. Physiol., A. in press.

El-Mossalami, M. K. and Emara, M. T. (1999). Safety and quality of fresh water crayfish Procambarus clarkii in the River Nile. Nahrung., 43 (2): 126128.

Emam,W. M.and khalil, M.T. (1995). Population dynamics and stock assessment of the newly introduced crayfish Procambarus clarkii in the River Nile, Proc. Zool. Soc. A.R. Egypt., 26 :131-143 .

Fernandes, M.A.S.; Mendonça, M.I.R.; Marques, J.C. and Madeira, V.M.C. (1995). Energy and nutrient storage capacity of Procambarus clarkii in the lower Mondego river valley (Portugal). Freshw. crayfish., 10: 98-104.

Habashy, M. M. (2004a). Survival and growth rates of Procambarus clarkii, Girard (Crustacea, Decapoda) under different temperature and salinity levels. J. Egypt. Ger. Soc. Zool., 43 (D): 135-146.

Hagerman, L.; Sondergaard, T.; Weile, K.; Hosie, D. and Uglow, R.F. (1990). Aspects of blood physiology and ammonia excretion in Nephrops norvegicus under hypoxia. Comp. Biochem. Physiol., A. 97: 51-55. 
Hammond, K. S.; Hollows, J. W.; Townsend, C. R. and Lokman, P. M. (2006). Effects of temperature and water calcium concentration on growth, survival and moulting of freshwater crayfish, Paranephrops zealandicus. Aquacult., 251 (2-4): 271-279.

Hubbard, D.M.; Robinson, E.H.; Brown, P.B. and Daniels, W.H. (1986). Optimum ratio of dietary protein to energy for red crayfish (Procambarus clarkii). Prog. Fish Cult.,48: 233-237.

Huner, J. V.; Könönen, H. and Lindqvist, O. V. (1990). Variation in body composition and exoskeleton mineralization as functions of the molt and reproductive cycles of the noble crayfish, Astacus astacus L. (Decapoda, Astacidae), from a pond in central Finland. Comp. Biochem. Physiol., A. 96 (1): 235-240.

Huner, J. V.; Lindqvist, O. V. and Könönen, H. (1988). Comparison of morphology and edible tissues of two important commercial crayfishes, the noble crayfish, Astacus astacus Linné, and the red swamp crayfish Procambarus clarkii (Girard) (Decapoda, Astacidae and Cambaridae). Aquacult., 68 (1): 45-57.

Ibrahim, A. M; Khalil, M. T. and Mubarak, F. M. (1995). On the feeding behaviour of the exotic crayfish Procambarus clarkii in Egypt and its prospects in the biocontrol of local vector snails. J. Union Arab Biol., Cairo. , 4 (A): 321-340.

Ibrahim, A. M; Khalil, M. T. and Mubarak, F. M. (1996). Ecological studies on the exotic crayfishes Procambarus clarkii (Girard, 1852) and $P$. zonangulus (Hobbs and Hobbs, 1990), in the River Nile Egypt. J. Egypt. Ger. Soc. Zool., 20 (D): 176-185.

Ingerson, T. I. and Geddes, M.C.(1995). Respiration and survival of yabbies, Cherax destructor Clark, during hypoxia and the effect of hypoxia on growth in laboratory. Freshwat. Crayfish., 10: 221-229.

Johnson Jr, W. B. and Avault Jr, J. W. (1982). Effects of poultry waste supplementation to rice-crayfish (Orysa sativa-Procambarus clarkii) culture ponds. Aquacult., 29 (1-2): 109-123. 
Jones, C. M. (1995). Effect of temperature on growth and survival of the tropical freshwater crayfish Cherax quadricarinatus (Von Martens) (Decapoda, Parastcidae). Freshwat. Crayfish., 8: 391-398.

Khan, S. and Nugegoda, D. (2007). Sensitivity of juvenile freshwater crayfish Cherax destructor (Decapoda: Parastacidae) to trace metals. Ecotoxicol. Environ. Saf. 68: 463-469.

Liu, H.; Avauit, J. W. and Medley, P. (1995). Toxicity of ammonia and nitrite to juvenile red claw crayfish Cherax quadricarinatus (Von Martens). Freshw. Crayfish, 10: 249-255.

Madigosky, S.R.;Alvarez- Hermández, X. and Glass, J.(1991). Lead, cadmium and aluminium accumulation in the red swamp crayfish Procambarus clarkii collected from roadside drainage ditches in Louisiana. Arch. Environ. Contam. Toxicol., 20 :253-258 .

Maranhao, P.; Marques, J. C. and Madeira, V. (1995). Copper concentrations in soft tissues of the red swamp crayfish Procambarus clarkii (Girard, 1852) after exposure to a range of dissolved copper concentrations. Freshw. Crayfish, 10: 282-286.

Mauro, N. A. and Thompson, C. (1984). Hypoxia adaptation in the crayfish Procambarus clarkii. Comp. Biochem. Physiol., A. 79 (1): 73-75.

Meyer, W.; Kretschmer, M.; Hoffmann, A. and Harisch, G. (1991). Biochemical and histochemical observations on effects of low-level heavy metal load (lead, cadmium) in different organ systems of the freshwater crayfish, Astacus astacus L. (Crustacea: Decapoda). Ecotoxicol. Environ. Saf.,21 (2): 137-156.

Millamena, O. M. and Pascual, F. P. (1990). Tissue lipid content and fatty acid composition of Penaus monodon Fabricius broodstock from the wild. J. World Aquac. Soc., 21: 116-121.

Mona, M. H; Geasa, N. M. Sh.; Sharshar, Kh. M. and Morsy, E. M. (2000). Chemical composition of fresh water crayfish Procambarus clarkii and its nutritive value. Egypt. J. Aquat. Biol \& Fish., 4 (1): 19-34. 
Mubarak, M. F. (1997). Ecological studies of the newly introduced fresh water crayfish Procambarus clarkii and its impact upon the diversity of zooplankton and bottom fauna in the River Nile, Egypt. M.Sc. Thesis. Institute of Environ. Studi. \& Res., Ain Shams Univ., Cairo, Egypt.

Mubarak, M. F. (2001). Fishery management of the exotic crayfish Procambarus clarkii in the irrigation canal system of the River Nile, Egypt. Ph. D. Thesis, Institute of Environ. Studi. \& Res., Ain Shams Univ., Cairo, Egypt.

Mwangi , S. M. and Alikhan, M. A.(1993). Cadmium and nickel uptake by tissues of Cambarus bartoni (Astacidae, Decapoda, Crustacea) effects on copper and zinc stores. Elsevier Sci. water Res. 27(5): 921-927.

Payette, A. L. and Mc Gaw, I. J. (2003 ). Thermoregulatory behavior of the crayfish Procambarus clarkii in a burrow environment. Comp. Biochem. Physiol., A (136):539-556.

Rainbow, P. S. (1997). Ecophysiology of trace metals uptake in crustaceans. Estuar. Coast Shelf Sci., 44:169-175.

Rainbow, P. S. (2002). Trace metal concentration in aquatic invertebrates: why and so what? Environ. Pollut., 120: 497-507.

Rizkalla, E. and Abo-Donia, M. A. (1996). Some industrial heavy metal pollutants in River Nile and their effect on biological and non biological inhabitants 1- Essential heavy metals. Egypt. J. Agric. Res., 74 (1): 225-237.

Rodríguez-González, H.; García-Ulloa, M.; Hernández-Llamas, A. and Villarreal, H. (2006). Effect of dietary protein level on spawning and egg quality of redclaw crayfish Cherax quadricarinatus. Aquacult., 257 (1-4): 412-419.

Roesijadi, G. (1992). Metallothioneins in metal regulation and toxicity in aquatic animals. Aquat. Toxicol., 22 (2): 81-114. 
Roesijadi, G. (1994). Metallothionein induction as a measure of response to metal exposure in aquatic animals. Environ. Health Perspect., 102: 91-95.

Rosa, R. A. and Nunes, M. L. (2003a). Biochemical composition of deep-sea decapod crustaceans with two different benthic life strategies off the Portuguese south coast. Deep-Sea Res., 50 (1): 119-130.

Rosa, R. A. and Nunes, M. L. (2003b). Changes in organ indices and lipid dynamics during the reproductive cycle of Aristeus antennatus, Parapenaeus longirostris and Nephrops norvegicus (Crustacea: Decapoda) females from the south Portuguese coast. Crustaceana, 75: 1095-1105.

Rouse, D. B. and Yeh, H. S. (1995). Factors influencing spawning of Cherax quadrinatus in indoor hatcheries. Freshw. Crayfish, 10:605-610 .

Rowe, C. L.; Hopkins, W. A.; Zehnder, C. and Congdon, J. D. (2001). Metabolic costs incurred by crayfish (Procambarus acutus) in a trace elementpolluted habitat: further evidence of similar responses among diverse taxonomic groups. Comp. Biochem. Physiol., C. 129 (3): 275-283.

Saad, A. A. and Emam, W. M. (1998). Field and semifield studies on the crayfish Procambarus clarkii in Egypt. J. Aquat. Biol.\& Fish., 2 (4): 331-344.

Sayed, M. M. (2002). Biological studies on the crayfish Procambarus clarkii in the River Nile. M. Sc. Thesis., Zool. Dept., Fac. of Sci., Ain Shams Univ., Egypt.

Schirf, V. R.; Turner, P.; Selby, L.; Hannapel, C.; De La Cruz, P. and Dehn, P. F. (1987). Nutritional status and energy metabolism of crayfish (Procambarus clarkii, Girard) muscle and hepatopancreas. Comp. Biochem. Physiol., A. 88 (3): 383-386.

Shakir, S. H. and Ibrahim, A. M. (1998). Population dynamics and distribution of the newly introduced crawfish Procambarus clarkii in Egypt using geographic information system (GIS). Egypt. J. Aquat. Biol. \& Fish., 2 (4): 537-551. 
Silva-Castiglioni, D.; Dutra, B. K; Oliveira, G. T. and Buckup, G. B. (2007). Seasonal variations in the intermediate metabolism of Parastacus varicosus (Crustacea, Decapoda, Parastacidae). Comp. Biochem. Physiol., A. 148 (1): 204-213.

Sokolov, W. (1966). Water content in tissues of desert animals. Nat., 211: 545.

Teshima, S. and Kanazawa, A. (1983). Variation in lipid composition during the ovarian maturation of the prawn. Bull. Jpn. Soc. Sci. Fish., 49: 957962.

Tietz, N. W; Fierech, E. A; Berger, S.; Blanke, E. W.; Forsman, D. T.; Freier, E. F. and Carawey, W. T. (1970). Fundamentals of clinical chemistry. W. \& B. Sounders Comp., London.

Tolba, M. R. (1999). The red swamp crayfish Procambarus clarkii (Decapoda: Cambaridae) as bio-indicator for total water quality including $\mathrm{Cu}$ and Cd pollution. Egypt. J. Aquat. Biol \& Fish., 3 (1): 59-71.

Torreblanca, A.; Ramo, J. D. and Diaz-Mayans, J. (1992). Changes in biochemical composition of gills, hepatopancreas, and muscle of the red crayfish Procambarus clarkii (girard) after sub- lethal exposure to mercury. Comp. Biochem. Physiol., C. 102 (2): 247-252.

Vinagre, A. S.; Amaral, A. P. N.; Ribarcki, F. P.; Silveira, E. F. and Pèrico, E. (2007). Seasonal variation of energy metabolism in ghost crab Ocypode quadrata at Siriù Beach (Brazil). Comp. Biochem. Physiol., A. 146 (4): 514-519.

Wooton, L. D. P. (1964):. Microanalysis in medical biochemistry. $7^{\text {th }}$ ed., Chruchill., London, Basal, Karger, 264pp.

Zanatto, P.; Wheatly, M. G.; Reiber, C. L.; Cannon, A. J. and Jalles-Filho, E. L.(2004). Allometric relationship of postmolt net ion uptake; ventilation and circulation in the freshwater crayfish Procambarus clarkii, intraspesific scaling. Physiol. Biochem. Zool., 77:275-284. 